$x \oplus 7 \frac{1}{3}$

\title{
AN EFFICIENT MODIFICATION OF \\ EULER-MACLAURIN'S FORMULA
}

(Trans. of JSCE, No. 24, April. 1955)

\author{
Dr. Eng., Bennosuke TANIMOTO, C.E. Member*
}

\begin{abstract}
Synopsis. Euler-Maclaurin's formula has widely been used for numerical integration, but at times successive derivatives involved are labourious to work out. A device will then be developed that the third derivative term is missing. Simple applications of the new rule will be given satisfactorily.
\end{abstract}

1. The well-known Euler-Maclaurin's formula for numerical evaluation of the definite integral

$$
I=\int_{a}^{b} f(x) d x
$$

takes the form

$$
\begin{aligned}
I=h\left(\frac{1}{2} f_{0}\right. & \left.+f_{1}+f_{2}+\cdots \cdots+f_{n-1}+\frac{1}{2} f_{n}\right) \\
& -\frac{h^{2}}{12}\left(f_{n}^{\prime}-f_{0}^{\prime}\right)+\frac{h^{4}}{720}\left(f_{n}^{\prime \prime \prime}-f_{0}^{\prime \prime \prime}\right)-\frac{h^{6}}{30240}\left(f_{n}^{V}-f_{0}^{V}\right)+\cdots \cdots .
\end{aligned}
$$

This formula was given as early as in 1730-1740, and has widely been used for evaluating the integral (1), when analytical method of its evaluation is difficult or impossible ${ }^{1}$.

The first term in the right side of the formula (2) above, which is included in the first parentheses, may be taken as a rough approximation by trapezoidal rule to the integral ( 1 ), and the succeeding terms involving derivatives may consequently be considered as the correction to the rough approximation.

An alternative form similar to (2) will be obtained by commencing with the assumption

$$
I=\frac{h}{3}\left(f_{0}+4 f_{1}+2 f_{2}+4 f_{3}+2 f_{4}+\cdots \cdots+2 f_{2 n-2}+4 f_{2 n-1}+f_{2 n}\right)+T,
$$

in which the first term in parentheses is an approximation to the integral due to Simpson's rule, which is more accurate than that due to trapezoidal rule, and $T$ denotes certain correction-terms, the analytical form of which will be found later. With the aid of this formula, the third derivative term in (2) can be eliminated, and the formula so formed will in general be preferable to (2), when much labour is needed to work out higher derivatives of the integrand $f(x)$, which will at times be encountered in engineering and physical problems.

2. The function $f(x)$ is not known, but without loss of generality we may put ${ }^{2 y}$

$$
f(x)=e^{c x},
$$

$c$ being constant independent of the variable $x$. By a simple analytical process we have

$$
I=\int_{a}^{b} f(x) d x=\int_{a}^{a+2 n \hbar} e^{c x} d x=\frac{e^{c a}}{c}\left(e^{2 n c h}-1\right) ;
$$

and on the other hand, repeated application of Simpson's rule affords

$$
\begin{aligned}
I=\int_{a}^{a+2 n h} e^{c x} d x=\frac{h}{3}\left[e^{c a}\right. & +4 e^{c(a+h)}+2 e^{c(a+2 h)}+\cdots \cdots+2 e^{c(a+2 n-2 h)} \\
& \left.+4 e^{c(a+\overline{2 n-1} h)}+e^{c(a+2 n h)}\right]+T
\end{aligned}
$$

* Professor of Civil Engineering, Faculty of Engineering, Shinshu University, Nagano, Japan. 


$$
=\frac{h}{3} e^{c a}\left[\left(e^{2 n c h}-1\right)+2\left(2 e^{c h}+1\right)\left(1+e^{2 c h}+e^{4 c h}+\cdots \cdots+e^{(2 n-2) c h}\right)\right]+T,
$$

$T$ being correction-terms as before. Now we know the sum

$$
1+e^{2 c h}+e^{4 c h}+\cdots \cdots+e^{(2 n-2) c h}=\frac{e^{2 n c h}-1}{e^{2 c h}-1},
$$

so that (4) becomes

$$
I=e^{c a}\left(e^{2 n c h}-1\right) \frac{2 h}{3}\left(\frac{1}{2}+\frac{2 e^{c h}+1}{e^{2 c h}-1}\right)+T .
$$

Comparing this with (3), we have

$$
T=-e^{c a}\left(e^{2 n c h}-1\right) \frac{2 h}{3}\left(-\frac{3}{2 c h}+\frac{1}{2}+\frac{2 e^{c h}+1}{e^{2 c h}-1}\right) .
$$

The last factor in parentheses may be expanded into an ascending power series of $c h$, for

$$
2 e^{c h}+1=3+\frac{2 c h}{1 !}+\frac{2(c h)^{2}}{2 !}+\frac{2(c h)^{3}}{3 !}+\cdots \cdots,
$$

and

$$
\frac{1}{e^{2 c h}-1}=\frac{1}{2 c h}-\frac{1}{2}+\frac{B_{2}}{2 !} 2 c h+\frac{B_{4}}{4 !}(2 c h)^{3}+\frac{B_{6}}{6 !}(2 c h)^{5}+\cdots \cdots,
$$

where $B_{2}, B_{4}, B_{6}, \cdots \cdots$ represent Bernoullian numbers; viz.

$$
B_{2}=\frac{1}{6}, \quad B_{4}=-\frac{1}{30}, \quad B_{6}=\frac{1}{42},
$$

Hence $T$ for the time being becomes

$$
T=-e^{c a}\left(e^{2 n c h}-1\right)\left(\frac{c^{3} h^{4}}{180}+\frac{c^{5} h^{6}}{1512}+\cdots \cdots\right) .
$$

Since $f(x)$ takes the form $f(x)=e^{c x}$, we at once have

$$
f_{2 n}^{\prime \prime \prime}-f_{0}^{\prime \prime \prime}=c^{3} e^{c a}\left(e^{2 n c h}-1\right), \quad f_{2 n}^{V}-f_{0}^{V}=c^{5} e^{c a}\left(e^{2 n c h}-1\right),
$$

Thus we obtain

$$
\begin{aligned}
\int_{a}^{a+2 n h} f(x) d x= & \frac{h}{3}\left(f_{0}+4 f_{1}+2 f_{2}+\cdots \cdots+2 f_{2 n-2}+4 f_{2 n-1}+f_{2 n}\right) \\
& -\frac{h^{4}}{180}\left(f_{2 n}^{\prime \prime \prime}-f_{0}^{\prime \prime \prime}\right)-\frac{h^{6}}{1512}\left(f_{2 n}{ }^{V}-f_{0} V\right)-\cdots \cdots \cdots \cdots
\end{aligned}
$$

If further we eliminate third derivative terms between (5) and (2), in the latter $n$ being replaced by $2 n$, then we obtain

$$
\begin{aligned}
I=\frac{h}{15}\left(7 f_{0}+16\right. & \left.f_{1}+14 f_{2}+\cdots \cdots+14 f_{2 n-2}+16 f_{2 n-1}+7 f_{2 n}\right) \\
& -\frac{h^{2}}{15}\left(f_{2 n}{ }^{\prime}-f_{0}{ }^{\prime}\right)-\frac{h^{6}}{6300}\left(f_{2 n} V-f_{0} V\right)-\cdots \cdots \cdots \cdots .
\end{aligned}
$$

This is the required expression. When the fifth and higher derivative terms can be neglected as small, the formula last obtained will furnish a prompt and efficient means for evaluating the integral (1), since in this equation it is sufficient to compute only first derivative for the evaluation in question in virtue of the absence of the third derivative term. A slight inferiority in the new formula (6) is however to be noted as the numerical coefficients in (6) are less convergent than those in (2); but this would appear in most cases to be superseded by the present device that the third derivative term has been eliminated. Also, it is of practical importance to note that the rapid convergence of succeeding terms in (6) at times fails, if too rough value of the interval of divided parts, $h$, is selected, as is the case in the Euler-Maclaurin's formula (2).

3. It is sometimes requested to evaluate the integral of the form

$$
I=\int_{0}^{\infty} f(x) d x
$$


in which $f(x)$ tends to zero for large values of $x$. In such a case (6) reduces to

$$
\begin{aligned}
I=\frac{h}{15}\left(7 f_{0}+16 f_{1}+14 f_{2}+16\right. & \left.f_{3}+14 f_{4}+\cdots \cdots\right) \\
& +\frac{h^{2}}{15} f_{0}^{\prime}+\frac{h^{6}}{6300} f_{0} V+\cdots \cdots \cdots \cdots .
\end{aligned}
$$

It would not be so labourious to compute the integral (7), when the integrand $f(x)$ in (7) is of rapid convergence.

Duplicate use of (8) to the double definite integral ${ }^{3)}$

$$
I=\int_{0}^{\infty} \int_{0}^{\infty} f(x, y) d x d y
$$

will afford the result

$$
\begin{aligned}
I= & \frac{h k}{225}[7\{7 f(00)+16 f(10)+14 f(20)+\cdots \cdots\}+16\{7 f(01)+16 f(11) \\
& +14 f(21)+\cdots \cdots\}+14\{7 f(02)+16 f(12)+14 f(22)+\cdots \cdots\}+\cdots \cdots] \\
& +\frac{h k^{2}}{225}\left[7 f_{y}(00)+16 f_{y}(10)+14 f_{y}(20)+\cdots \cdots\right] \\
& +\frac{h^{2} k}{225}\left[7 f_{x}(00)+16 f_{x}(01)+14 f_{x}(02)+\cdots \cdots\right]+\frac{h^{2} k^{2}}{225} f_{x y}(00)+\cdots \cdots \cdots,
\end{aligned}
$$

where $f(00), f(10), f(20), \cdots \cdots f(01), f(02), \cdots \cdots$ denote values of the integrand $f(x, y)$ at mesh points $(00),(10),(20), \cdots \cdots(01),(02), \cdots \cdots$ as in Fig. 1 , and $f_{x}(00), f_{y}(00)$, etc. represent derivatives at $(00)$, etc. with respect to $x$ and $y$ in order.

Fig. 1 Arrangement of mesh points.
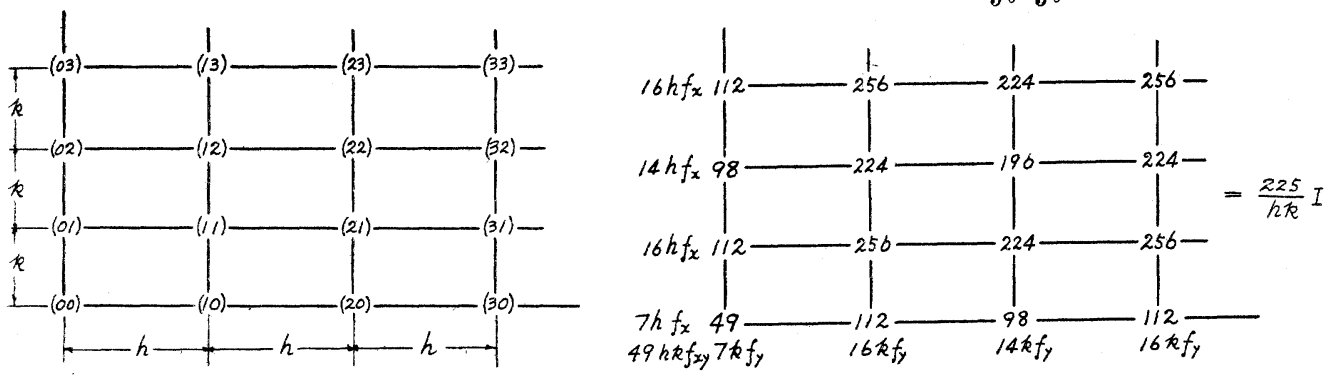

Equation (9) may also be expressed as in Fig. 2, which will be convenient for practical use.

4. As a simple application of (6), let us evaluate the definite integral

$$
I=\int_{0}^{\pi / 2} \sin x d x,=1 \text {. }
$$

Now we for convenience subdivide the domain of integration into 8 small parts of equal interval, so that $h=\pi / 16$. We then have, with (6) by curtailing the second and higher correction-terms,

$$
\begin{aligned}
I= & \frac{1}{15} \frac{\pi}{16}\left(7 \sin 0^{\circ}+16 \sin 11.25^{\circ}+14 \sin 22.5^{\circ}+16 \sin 33.75^{\circ}+14 \sin 45^{\circ}\right. \\
& \left.+16 \sin 56.25^{\circ}+14 \sin 67.5^{\circ}+16 \sin 78.75^{\circ}+7 \sin 90^{\circ}\right) \\
& -\frac{1}{15}\left(\frac{\pi}{16}\right)^{2}\left(\cos 90^{\circ}-\cos 0^{\circ}\right) \\
= & 0.01308997 \times(7 \times 0+16 \times 0.1950903+14 \times 0.3826834+16 \times 0.5555702 \\
& +14 \times 0.7071068+16 \times 0.8314696+14 \times 0.9238795+16 \times 0.9807853 \\
& +7 \times 1.0000000)+0.00257021=0.99742975+0.00257021=1.0000000 .
\end{aligned}
$$


In this computation only the first derivative is needed; nevertheless the result is accurate to the last digit. The reason of this accuracy can be found in the evaluation of the succeeding correction-term in (6), viz.

$$
-\frac{h^{6}}{6300}\left(f_{2 n} V-f_{0} V\right)=-\frac{1}{6300}\left(\frac{\pi}{16}\right)^{6}\left(\cos 90^{\circ}-\cos 0^{\prime}\right)=0.0000000091 \text {, }
$$

which does not affect the seventh digit of decimal.

On the other hand the prevailing Euler-Maclaurin's formula (2), when curtailing the second and higher correction-terms, gives

$$
\begin{aligned}
I= & \frac{\pi}{16} \times\left(\frac{1}{2} \times 0+0.1950903+0.3826834+0.5555702+0.7071068\right. \\
& \left.+0.8314696+0.9238795+0.9807853+\frac{1}{2} \times 1.0000000\right) \\
& -\frac{1}{12}\left(\frac{\pi}{16}\right)^{2}\left(\cos 90^{\circ}-\cos 0^{\circ}\right)=0.99678515+0.00321276=0.9999979 ;
\end{aligned}
$$

and only when the third derivative is taken into account, the result will become accurate. In this connection the well-known Simpson's rule will afford the value 1.0000082 , which is much less accurate than the two preceding results.

5. An example of (9) is to evaluate the double definite integral

$$
I=-\frac{4}{\pi^{2}} \int_{0}^{\infty} \int_{0}^{\infty} \frac{1+z}{x y} \sin x \sin y e^{-z} d x d y,
$$

in which $z^{2}=x^{2}+y^{2}$. We write

$$
f(x, y)=\frac{1+z}{x y} \sin x \sin y e^{-z}
$$

and then we have

$$
\begin{aligned}
f_{x}= & \left(\frac{1+z}{x y} \cos x-\frac{1+z+x^{2}}{x^{2} y} \sin x\right) \sin y e^{-z}, \\
f_{y}= & \left(\frac{1+z}{x y} \cos y-\frac{1+z+y^{2}}{x y^{2}} \sin y\right) \sin x e^{-z}, \\
f_{x y}= & \left(\frac{1+z}{x y} \cos x \cos y-\frac{1+z+y^{2}}{x y^{2}} \cos x \sin y\right. \\
& \left.-\frac{1+z+x^{2}}{x^{2} y} \sin x \cos y+\frac{z+z^{2}+z^{3}+x^{2} y^{2}}{x^{2} y^{2} z} \sin x \sin y\right) e^{-z} .
\end{aligned}
$$

It can be seen here that the last of these derivatives $f_{x y}$ vanishes at the origin where $(x, y)=(0,0)$, and in addition that both of $f_{y}$ along $x$-axis and $f_{x}$ along $y$-axis also vanish, so that no contribution to the present evaluation is furnished from the terms in $f_{x}, f_{y}$ and $f_{x y}$ of equation (9) or Fig. 2.

Now mesh-point values of the integrand $f(x, y)$ become as shown in Fig. 3 by taking $h=k=\pi / 4$. Then by multiplying corresponding values in Figs. 2 and 3 , and by summing up these products, we obtain

$$
I=-\frac{4}{\pi^{2}} \frac{1}{225}\left(\frac{\pi}{4}\right)^{2} \times 629.8=-0.700 .
$$

It is added however that this numerical example is a rather unsuitable application of the rule (9), since it is by chance missing terms involving derivatives as was stated above, and hence merely reduces to a linear combination of trapezoidal rule and Simpson's rule in two dimensions. 
Fig. 3. Mesh-point values of $f(x, y)=\frac{1+z}{x y} \sin x \sin y e^{-z}$.

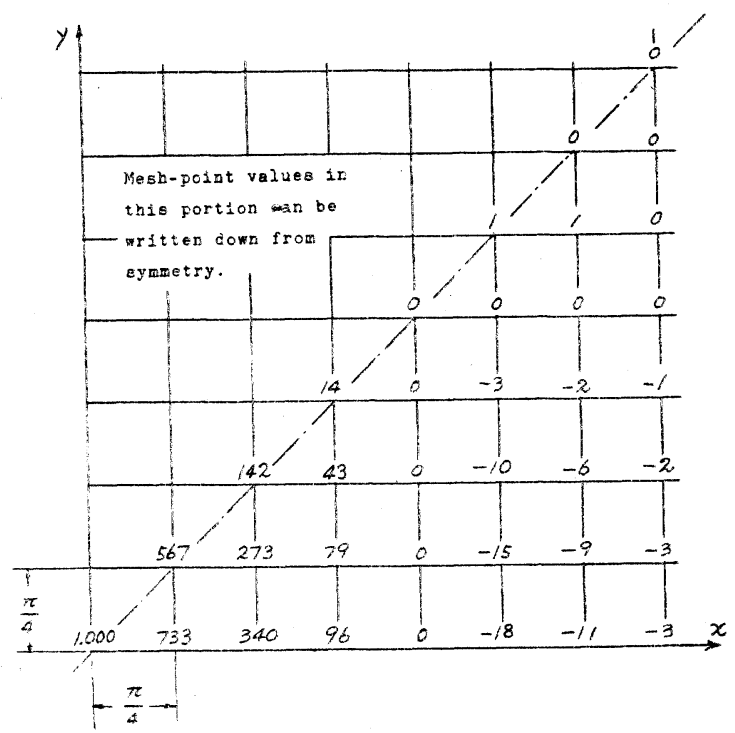

6. The necessity of evaluating the integral (10) above arose in connection with my analytical solution of a three-dimensional Boussinesq's problem in which a semiinfinite elastic solid body is pressed with a uniform load on its surface, and a number of expressions similar to (10) have been obtained as the solution of the generalized Boussinesq's problem that is capable of shearing forces as well as of normal pressure ${ }^{4)}$. In fact the value obtained in (12) is the magnitude of compressive stress at a point along the vertical axis and distant from the origin of coordinates by unit length, when the semi-infinite solid is pressed with a uniform load of unit stress distributed over a square whose side lengths are both 2 .

The value (12) above could be confirmed to be accurate by a further computation in which a finer mesh where $h=k=\pi / 8$ was adopted, and it gave the same value as (12). It is also in accordance with that computed otherwise by the late J. Kimura ${ }^{5}$, its analytical solution being due to A.E.H. Love ${ }^{6)}$ by means of integrating Boussinesq's potentials.

Acknowledgements. I should like to express my sincere thanks to Dr. Yutaka TANAKA, member of Japan Academy and professor emeritus of Tokyo University, for his affectionate supervision; and also to Dr. Tomoyasu YUKI, dean and professor in the Faculty of Engineering, Shinshu University, for giving some facilities for my work.

\section{References}

1) Whittaker \& Robinson: "The Calculus of Observations," London \& Glasgow, 1937, Chapter VII.

2) Loc. cit. 1), p. 135.

3) With regard to a numerical method on the double definite integral, derived from interpolation formluas, reference may be made to my work entitled "On the Mechanical Cubature," Journal of the Shinshu University, No. 3, 1954.

4) Oral report of the work was made at the 9th Annual Congress of the Japan Society of Civil Engineers at Sendai, May 1953 ; and it will be printed in the near future.

5) Bulletin of the Geotechnical Committee, Government Railways of Japan, No. 1 (June 1931) (in Japanese).

6) Philosophical Transactions, London, Series A, vol. 228 (1929). 